OPEN ACCESS

Edited by:

Anders Omsland,

Washington State University, USA

Reviewed by:

Jason A. Carlyon,

Virginia Commonwealth University

School of Medicine, USA

Laszlo Kari,

National Institute of Allergy and

Infectious Diseases (NIH), USA

${ }^{*}$ Correspondence:

Isabelle Derré

id8m@virginia.edu

Received: 22 November 2016 Accepted: 27 February 2017

Published: 14 March 2017

Citation:

Han Y and Derré I (2017) A Co-infection Model System and the Use of Chimeric Proteins to Study

Chlamydia Inclusion Proteins Interaction

Front. Cell. Infect. Microbiol. 7:79. doi: 10.3389/fcimb.2017.00079

\section{A Co-infection Model System and the Use of Chimeric Proteins to Study Chlamydia Inclusion Proteins Interaction}

\author{
Ying $\operatorname{Han}^{1}$ and Isabelle Derrée ${ }^{2 *}$ \\ ${ }^{1}$ Department of Molecular Biophysics and Biochemistry, Yale University, New Haven, CT, USA, ${ }^{2}$ Department of Microbiology, \\ Immunology and Cancer Biology, University of Virginia, Charlottesville, VA, USA
}

Chlamydia trachomatis is an obligate intracellular bacterium associated with trachoma and sexually transmitted diseases. During its intracellular developmental cycle, Chlamydia resides in a membrane bound compartment called the inclusion. A subset of Type III secreted effectors, the inclusion membrane proteins (Inc), are inserted into the inclusion membrane. Inc proteins are strategically positioned to promote inclusion interaction with host factors and organelles, a process required for bacterial replication, but little is known about Inc proteins function or host interacting partners. Moreover, it is unclear whether each Inc protein has a distinct function or if a subset of Inc proteins interacts with one another to perform their function. Here, we used IncD as a model to investigate Inc/Inc interaction in the context of Inc protein expression in C. trachomatis. We developed a co-infection model system to display different tagged Inc proteins on the surface of the same inclusion. We also designed chimeric Inc proteins to delineate domains important for interaction. We showed that IncD can self-interact and that the full-length protein is required for dimerization and/or oligomerization. Altogether our approach can be generalized to any Inc protein and will help to characterize the molecular mechanisms by which Chlamydia Inc proteins interact with themselves and/or host factors, eventually leading to a better understanding of $C$. trachomatis interaction with the mammalian host.

Keywords: Chlamydia, inclusion membrane protein, IncD, IncE, chimeric Inc protein, homo- and heterotypic interaction

\section{INTRODUCTION}

Chlamydia trachomatis is an obligate intracellular bacterial pathogen responsible for the most common preventable blindness from infectious origin and is the leading cause of sexually transmitted infection of bacterial origin (Schachter, 1999). The ocular and genital tract epithelia are the primary sites of infection. After entry, C. trachomatis resides in a membrane-bound compartment, called the inclusion (Moulder, 1991). Within the lumen of the inclusion, the bacteria undergo a complex developmental cycle alternating between infectious and replicative forms. During co-evolution with the mammalian host, the $C$. trachomatis genome was reduced to about 900 open reading frames (ORF) (Stephens et al., 1998) and C. trachomatis has evolved sophisticated mechanisms to hijack cellular organelles and manipulate cellular pathways to acquire essential 
nutrients (Elwell et al., 2016). Central to these processes is a bacterial Type III secretion system (T3SS), which allows for the translocation of bacterial effectors from the bacterial cytosol into the host cell (Mueller et al., 2014). One family of C. trachomatis T3SS effectors, are inserted into the inclusion membrane and referred to as the inclusion membrane proteins (Inc) (Dehoux et al., 2011; Lutter et al., 2012; Moore and Ouellette, 2014).

Chlamydia inclusion membrane proteins are characterized by a large bilobed hydrophobic domain containing at least 50 amino acids (Bannantine et al., 2000), and amino- and carboxyterminal tails that are presumably exposed on the cytosolic surface of the inclusion membrane. Based on the presence of the bilobed hydrophobic domain, the C. trachomatis genome is predicted to encode up to 60 Inc proteins. The inclusion membrane localization of a large number of predicted $C$. trachomatis Inc proteins has been confirmed using primary antibodies (Li et al., 2008) or expression of tagged proteins from the bacteria (Weber et al., 2015). These Type III secreted effectors are unique to Chlamydia and are strategically positioned to mediate the interaction of the inclusion with cellular factors and organelles.

Only a few C. trachomatis Inc proteins have been assigned a host interacting partner and/or a function. IncA is involved in homotypic fusion of inclusions (Hackstadt et al., 1999). IncG interacts with 14-3-3ß (Scidmore and Hackstadt, 2001) and CT229 with Rab4 (Rzomp et al., 2006). IncD interacts with and recruits the ceramide transfer protein, CERT, to ERinclusion membrane contact sites (Derre et al., 2011; Agaisse and Derre, 2014). CT228 interacts with the myosin phosphatase target subunit 1 MYPT1 and regulates the mechanisms by which C. trachomatis egresses from the host cell (Lutter et al., 2013). CT850 interacts with the dynein light chain DYNLT1 (Mital et al., 2015). InaC (CT813/CTL0184) mediates the recruitment of $14-3-3 \beta, 14-3-3 \epsilon$, and ARF1 to the inclusion membrane and is involved in actin assembly and Golgi positioning around the inclusion (Kokes et al., 2015). IncE binds to the sorting nexins SNX5 and SNX6, and recruits these retromer complex components to the inclusion, resulting in inclusion membrane tubulation (Aeberhard et al., 2015; Mirrashidi et al., 2015). Mirrashidi et al. have also identified putative mammalian interacting partners for nearly 40 C. trachomatis Inc proteins (Mirrashidi et al., 2015). The study was performed with the Inc proteins overexpressed in mammalian cells, so these interactions remain to be validated during infection, but the study detected IncD/CERT and CT228/MYPT1 interactions, suggesting that this human/Inc interactome is a solid foundation to further investigate the function of $C$. trachomatis Inc proteins.

It is unclear whether each Inc protein has a distinct function or if a subset of Inc proteins can act in concert, potentially through direct interaction. Some Inc proteins are evenly distributed on the surface of the inclusion membrane, while others are concentrated in microdomains. This is best illustrated with IncB, Inc101, Inc222, and Inc850, which co-localize to discrete punctae of the inclusion membrane (Mital et al., 2010). Inc222 and Inc850 were shown to interact, suggesting that Inc proteins could form stable complexes with one another. The homo- or heterotypic interaction of Inc proteins was independently investigated using a bacterial two-hybrid system (Gauliard et al., 2015). Inc222 and Inc850 interaction was observed using this experimental set up, and the data also suggested that IncD interacted with itself. In addition, IncA, IncG, IncF, CT229 (CTL0481), CT058 (CTL0314), and CT222 (CTL0475) were identified as potential interacting partners of IncD. While the CT058- and CT222-IncD interactions were reciprocal, the IncA-, IncG-, IncF-, and CT229IncD interactions were unidirectional with this subset of Inc being able to interact with $\mathrm{IncD}$, but IncD did not interact with these proteins.

Here we have developed a system to test Inc/Inc homoand heterotypic interactions in the context of C. trachomatis infection and to identify domains that support these interactions. Our system relies on the homotypic fusion properties of C. trachomatis inclusions, on the co-infection with C. trachomatis strains expressing Inc proteins fused to different tags and on the expression of chimeric Inc proteins.

\section{MATERIALS AND METHODS}

\section{Ethics Statement}

All genetic manipulations and containment work were approved by the UVA Biosafety Committee and are in compliance with the section III-D-1-a of the National Institutes of Health guidelines for research involving recombinant DNA molecules.

\section{Cell Lines and Bacterial Strains}

HeLa cells were obtained from ATCC (CCL-2) and cultured at $37^{\circ} \mathrm{C}$ with $5 \% \mathrm{CO}_{2}$ in DMEM high glucose (Invitrogen) supplemented with 10\% heat inactivated FBS (Invitrogen). C. trachomatis Lymphogranuloma venereum, Type II were obtained from ATCC (L2/434/Bu VR-902B). Chlamydia propagation and infection were performed as previously described (Derre et al., 2007).

\section{Plasmid Construction}

Restriction enzymes and T4 DNA ligase were obtained from New England Biolabs (Ipswich, MA). PCR was performed using Herculase DNA polymerase (Stratagene). PCR primers were obtained from Integrated DNA Technologies. All the plasmids used in this study are derivatives of p2TK2-SW2 mCh(Gro) (Agaisse and Derre, 2014). They express mCherry under the control of the groESL operon promoter and terminator, the TetR repressor and the indicated Inc under the control of the tet $A$ gene promoter and incDEFG operon terminator. The p2TK2-SW2 mCh(Gro) Tet IncD-, IncE-, and IncG-3xFLAG plasmids were described previously (Agaisse and Derre, 2014; Mirrashidi et al., 2015). p2TK2-SW2 mCh(Gro) Tet IncD-Myc, CTL0314-3xFLAG, CTL0475-3xFLAG, or IncD/IncE-3xFLAG chimera were constructed similarly using the primers listed in Supplementary Table S1.

\section{C. trachomatis Transformation}

Our calcium-based transformation protocol was adapted from Wang et al. (2011) and is described in Agaisse and Derre (2013). 


\section{Immunoblotting}

Protein samples were separated by SDS-PAGE and transferred to nitrocellulose membranes. The membranes were blocked for $1 \mathrm{~h}$ at room temperature in 1xPBS containing $0.05 \%$ Tween and 5\% Fat-free milk. Primary and HRP-conjugated secondary antibobies were diluted in 1xPBS containing $0.05 \%$ Tween and $5 \%$ Fat-free milk and respectively incubated over-night at $4{ }^{\circ} \mathrm{C}$ and $1 \mathrm{~h}$ at room temperature. Proteins were detected using the Amersham ECL western blotting detection reagent as per manufacturer recommendation and a Biorad ChemiDoc imaging system.

\section{Immunofluorescence and Microscopy}

At the indicated times, HeLa cells seeded onto glass coverslips were fixed for $30 \mathrm{~min}$ in PBS containing $4 \%$ paraformaldehyde. Immunostainings were performed at room temperature. Antibodies were diluted in PBS containing $0.1 \%$ BSA and $0.1 \%$ Triton X-100. Samples were washed with PBS and examined under an epifluorescence or spinning disc confocal microscope.

\section{Antibodies}

The following primary antibodies were used: Mouse monoclonal anti-FLAG [1:1,000 (IF), 1:20,000 (WB), Sigma], mouse monoclonal anti-Myc [1:1,000 (IF), $1: 1,000$ (WB), Cell Signaling], rabbit polyclonal anti-Actin (1:1,000, Sigma), rabbit polyclonal anti-tRFP (1:2,000, Evrogen), and rabbit polyclonal anti-IncA (1:200, kind gift from T. Hackstadt, Rocky Mountain Laboratories). The following secondary antibodies were used: Peroxidase-conjugated goat anti-rabbit IgG (1:10,000, Jackson ImmunoResearch), peroxidase-conjugated goat anti-mouse IgG (1:10,000, Jackson ImmunoResearch), goat anti-mouse AlexaFluor 488 or 514 (IF: 1:1,000, Molecular Probes), and goat anti-rabbit Pacific Blue (IF: 1:1,000, Molecular Probes).

\section{Co-infection and Immunofluorescence Analysis of Co-infected Cells}

Each strain was used at an MOI of 5 to ensure that each eukaryotic cell would receive at least one of each bacterium leading to a mixed bacterial population in each inclusion and the cellular localization of the 3xFLAG- and Myc-tagged Inc proteins was analyzed by immunofluorescence. In our original experimental set up, the samples were co-stained with a rabbit anti-FLAG and a mouse anti-Myc. However, the rabbit antiFLAG led to uneven staining of the inclusions (even at higher concentration). Duplicate coverslips from the same co-infection were therefore stained with mouse anti-FLAG or mouse antiMyc antibodies. Although not ideal, the MOI were carefully optimized so that, for each co-immunoprecipitation experiment, $100 \%$ of the inclusions were positive for both FLAG and Myc. The samples were not analyzed if it was not the case.

\section{Co-immunoprecipitation}

$8.10^{5} \mathrm{HeLa}$ cells plated in 6-well tissue culture dishes and infected with the indicated C. trachomatis strains for $24 \mathrm{~h}$ were washed once with 1x PBS and lysed for $20 \mathrm{~min}$ in $300 \mu \mathrm{l}$ of lysis buffer [20 mM Tris pH7.5, $150 \mathrm{mM} \mathrm{NaCl}, 2$ mM EDTA, 1\%Triton X100, $1 \mathrm{mM}$ PMSF and protease inhibitor cocktail (Roche)]. The lysates were centrifuge at $13,000 \mathrm{rpm}$ for $10 \mathrm{~min}$. An aliquot of the clarified lysate was collected (Lysate). The clarified lysates were incubated for $2 \mathrm{~h}$ in the presence of $10 \mu \mathrm{l}$ of anti-FLAG M2 agarose beads (Sigma). The beads were washed three times (20 mM Tris pH7.5, $150 \mathrm{mM} \mathrm{NaCl}, 2$ mM EDTA, 1\%Triton X-100) and the bound proteins were eluted with $15 \mu \mathrm{l}$ of elution buffer [20 mM Tris pH7.5, $150 \mathrm{mM} \mathrm{NaCl,} 2 \mathrm{mM}$ EDTA, $100 \mu \mathrm{g} / \mathrm{ml}$ 3XFLAG peptide (Sigma)]. Ten microliters of the eluted fraction was collected (IP). All steps were conducted at $4^{\circ} \mathrm{C}$.

\section{Reproducibility}

Each experiment was performed at least three times. Representative results are shown.

\section{RESULTS}

To investigate IncD interaction with a subset of Inc proteins in the context of $C$. trachomatis infection, we have generated $C$. trachomatis strains co-expressing mCherry under a constitutive promoter and IncD-3xFLAG, IncD-Myc, IncE-3xFLAG, IncG3xFLAG, CTL0314-3xFLAG (CT058), or CTL0475-3xFLAG (CT222) under the control of the anhydrotetracycline (aTc) inducible promoter. IncG, CTL0314 and CTL0475 were chosen based on their potential ability to interact with IncD (Gauliard et al., 2015) and IncE was included as a negative control.

To verify the expression of the constructs, HeLa cells were infected with the above listed C. trachomatis strains in the absence or in the presence of aTc and the corresponding cell lysates were analyzed by western-blot (Figure 1A). Detection of actin and mCherry respectively confirmed equal cell and bacterial number in the absence $(-\mathrm{aTc})$ or presence of $\mathrm{aTc}(+\mathrm{aTc})$. Anti-FLAG antibodies were used to detect the respective 3xFLAG tagged Inc proteins and anti-Myc antibodies were used to detect IncDMyc. As expected, in the absence of inducer the proteins were not expressed (Figure 1A, -aTc), however a signal corresponding to the expected molecular weight of the respective proteins was observed in the presence of aTc (Figure 1A, +aTc).

In addition, the inclusion membrane localization of the above listed 3xFLAG- or Myc-tagged Inc proteins was analyzed by immuno-fluorescence. For this purpose, HeLa cells infected with the respective Chlamydia strains in the absence or in the presence of aTc were fixed $24 \mathrm{~h}$ post-infection and stained with antibodies against the FLAG or Myc tag. The respective Inc proteins were not detected in the absence of inducer (not shown). However, in the presence of aTc, $100 \%$ of the inclusions were positive for the respective constructs and the pattern indicated that, as previously shown for IncD-, IncE-, and IncG-3xFLAG (Agaisse and Derre, 2014; Mirrashidi et al., 2015), all constructs localized to the inclusion membrane (Figure 1B). The inclusion localization of CTL0314-3xFLAG and CTL0475-3xFLAG was further confirmed by co-staining of the inclusions with an antiIncA antibody (Supplementary Figure 1).

To test the potential interaction between IncD and a subset of Inc proteins during C. trachomatis intracellular developmental cycle, we took advantage of the homotypic fusion properties of the C. trachomatis inclusions (Ridderhof and Barnes, 1989; Agaisse and Derre, 2013). HeLa cells were co-infected with two 
A

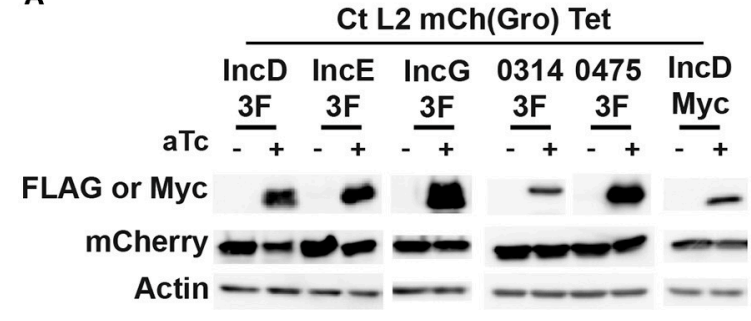

B
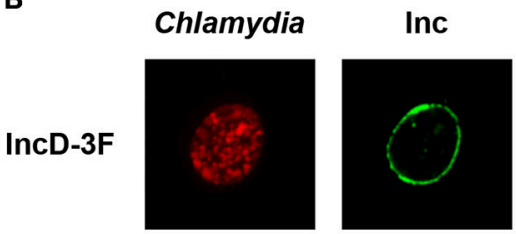

Merge

IncE-3F
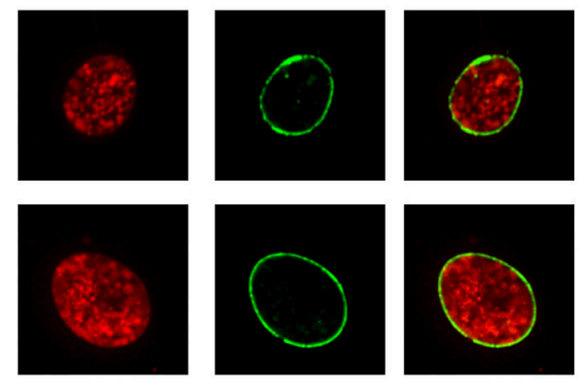

C
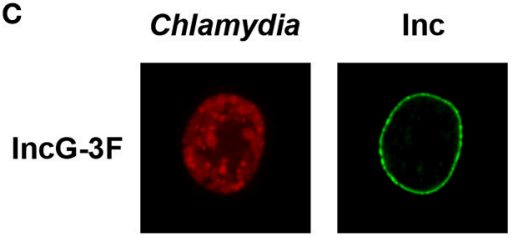

Merge
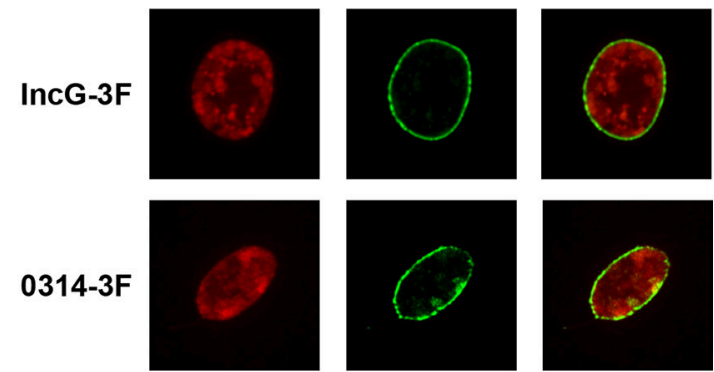

0475-3F
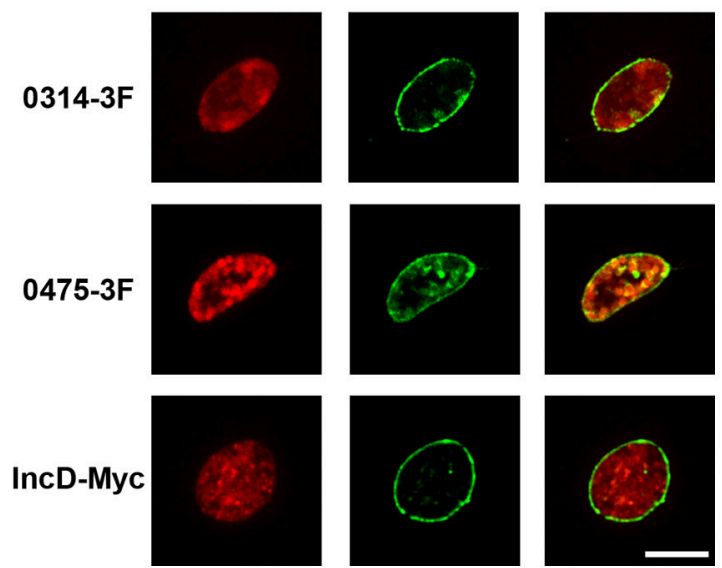

FIGURE 1 | Expression and inclusion localization of the subset of inclusion membrane proteins used in this study. (A) Immuno-blot of cell lysates from HeLa cells infected with strains of $C$. trachomatis expressing mCherry constitutively and IncD-, IncE-, IncG-, CTL0314-, CTL0475-3xFLAG (3F), or IncD-Myc under the control of the aTc inducible promoter. The cells were infected for $24 \mathrm{~h}$ in the absence (-aTc) or in the presence (+aTc) of $2 \mathrm{ng} / \mathrm{ml}$ aTc and immuno-blot of the corresponding lysates were probed using antibodies against FLAG or Myc, mCherry, and Actin. (B,C) Confocal micrographs of inclusions of the C. trachomatis strains listed in (A). The cells were infected in the presence of $2 \mathrm{ng} / \mathrm{ml}$ aTc, fixed $24 \mathrm{~h}$ post-infection, immunostained with anti-FLAG or anti-Myc antibodies and imaged using a confocal microscope. A single plane crossing the middle of the inclusion is shown. The left panels correspond to the bacteria (Chlamydia, red) and the middle panels to the 3xFLAG or Myc signal of the Inc constructs (Inc, green). The merge is shown on the right. Scale bar: $10 \mu \mathrm{m}$.

C. trachomatis strains expressing either IncE-3xFLAG or IncDMyc. Each strain was used at an MOI of 5 to ensure that each eukaryotic cell would receive at least one of each bacterium leading to a mixed bacterial population in each inclusion. The cellular localization of the IncE-3xFLAG and IncD-Myc proteins was analyzed by immunofluorescence (Figure 2). Close to $100 \%$ of the inclusions were positive for FLAG (Figure 2, Top Panels) or Myc (Figure 2, Bottom Panels) when the samples were immuno-labeled with one or the other antibody. Altogether, this result confirmed that the co-infection method allows for the insertion of different Inc proteins into the same inclusion membrane when the proteins are produced by different strains.

To investigate if IncD could interact with a subset of Inc proteins, including itself, HeLa cells were co-infected with the IncD-Myc expressing strain and a strain that expressed IncE-, IncG-, CTL0314-, CTL0475-, or IncD-3xFLAG, using the experimental set up described in Figure 2. For each condition, we confirmed that nearly $100 \%$ of the inclusions were positive for both markers (not shown). The samples were subjected to co-immuno-precipitation and the results were analyzed by western-blot (Figure 3). IncE-, IncG-, CTL0314, CTL0475-, and IncD-3xFLAG were expressed and efficiently immuno-precipitated with the anti-FLAG conjugated beads. IncD-Myc did not co-immuno-precipitate with IncE-, IncG, CTL0314-, or CTL0475-3xFLAG, suggesting that IncD does not interact with these Inc proteins. However, IncD-Myc did co-immuno-precipitate with IncD-3xFLAG (Figure 3, last lane), showing that IncD interacts with itself.

We next investigated whether the IncD/IncD interaction was occurring when the proteins were inserted into the inclusion membrane or "in vitro" after the cell lysates were prepared and processed for immuno-precipitation. To address this question, we compared IncD self-interaction as described above, or after collecting and co-incubating cell lysates from cells that were singly infected with either the IncD-3xFLAG strain or the IncDMyc strain. IncD self-interaction was observed upon co-infection (Figure 4, IP lane 3), however the IncD/IncD interaction was not detected when lysates from singly infected cells were combined prior immuno-precipitation (Figure 4, IP lane 4).

Altogether, these results indicate that, under our experimental set up, when C. trachomatis inclusion membrane proteins are inserted into the inclusion membrane, IncD interacts with itself but not with IncE, IncG, CTL0314, or CTL0475. Moreover, the IncD self-interaction was only observed when the IncD molecules were inserted into the same inclusion membrane.

We next sought to determine the IncD domain(s) mediating the IncD/IncD interaction. One possible approach would be to generate various internal, $\mathrm{N}$ - and $\mathrm{C}$-terminal truncated variant of IncD and assay for their self-interaction. However, Inc proteins secretion through the Chlamydia type III secretion system requires a $\mathrm{N}$-terminal secretion signal, that, if truncated, would prevent secretion. Moreover, the central hydrophobic domain, 


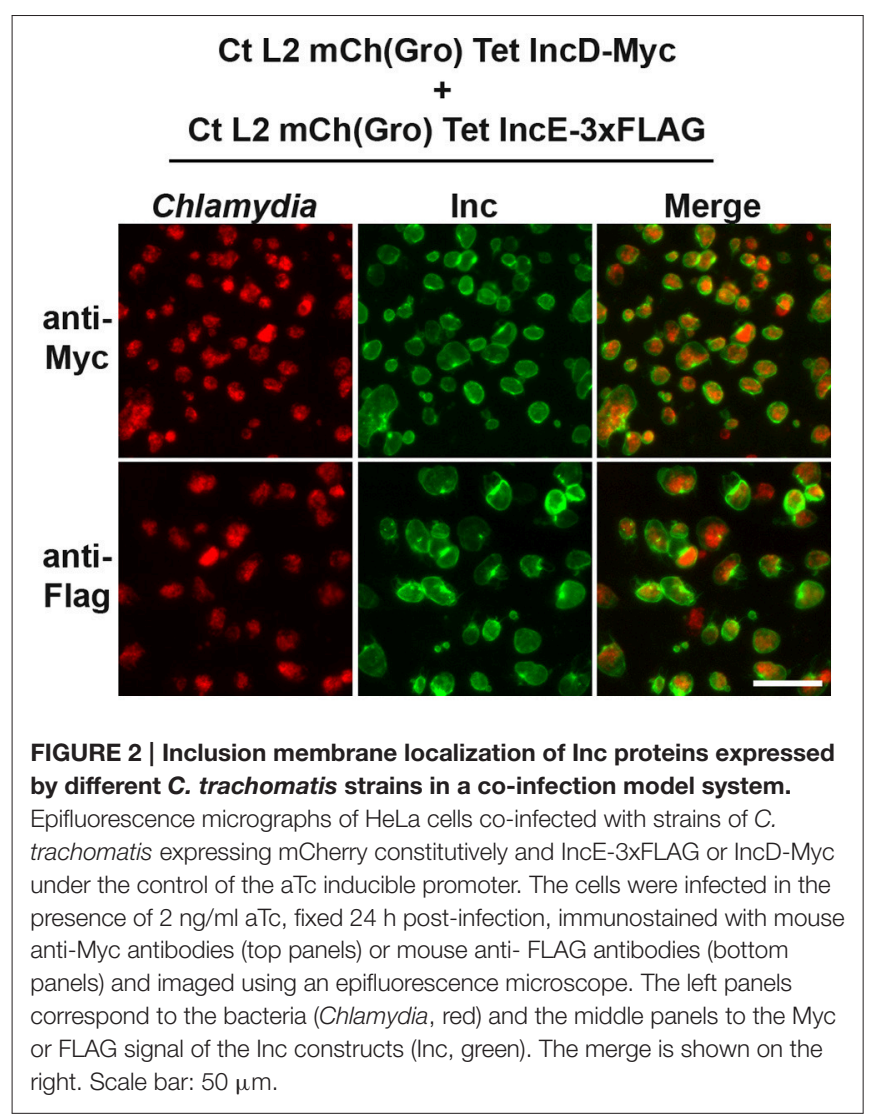

that is characteristic of Inc proteins, is most likely required for their insertion into the inclusion membrane, which would make its deletion incompatible with insertion of the corresponding Inc protein variant into the inclusion membrane.

To address these issues, we generated chimeric proteins between IncD and IncE. IncE was chosen because it does not interact with IncD (Figure 3) and IncD and IncE have similar molecular weight and display similar hydropathy profiles (Figure 5A). Based on IncD and IncE respective hydropathy profiles, each protein was divided into three domains: The $\mathrm{N}$ terminal domain (IncD: aa 1-39, IncE: aa 1-38), the hydrophobic domain (IncD: aa 40-91, IncE: aa 39-90), and the C-terminal domain (IncD: aa 92-146, IncE: aa 91-132). The following chimeric constructs were generated: IncDED, IncEED, IncDEE, IncEDE, IncDDE, and IncEDD where the first, second and third letter respectively correspond to the N-terminal, hydrophobic, or C-terminal domain of the respective Inc protein. The hydropathy profiles of the IncD/IncE chimeric proteins are shown in Figure 5A, where the IncD domains are indicated by the red circles. Each chimera was fused to a 3xFLAG tag and expressed under the control of the aTc inducible promoter into p2TK2-SW2mCh(Gro) and the corresponding plasmids were introduced into C. trachomatis. When HeLa cells were infected with the resulting $C$. trachomatis strains, mCherry was constitutively expressed and the IncD/IncE3xFLAG chimeric proteins were only detected in the presence of inducer (Figure 5B). The levels of expression of the chimera were

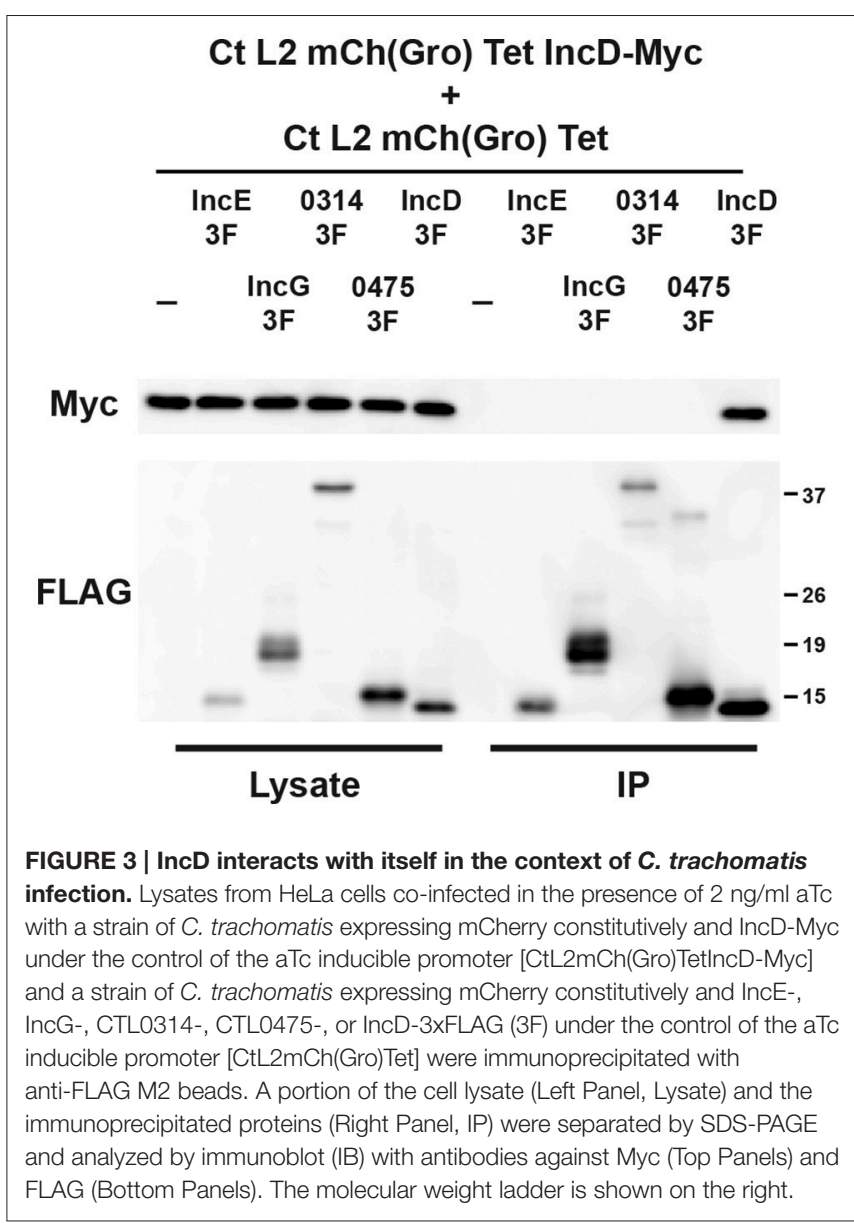

comparable to the one of IncD- or IncE-3xFLAG (Figure 1A) and the inclusion localization of the constructs was also confirmed by immunofluorescence (Figure 5C).

If the $\mathrm{N}$-terminal, the hydrophobic, or the $\mathrm{C}$-terminal domain of IncD is sufficient to mediate IncD self-interaction, we rationalized that this domain would promote $\mathrm{IncD} / \mathrm{IncD}$ E chimera interaction in our co-infection experimental set up. To test our hypothesis, HeLa cells were co-infected with C. trachomatis strains respectively expressing IncD-Myc and IncD-3xFLAG or IncD-Myc and one of the six IncD/IncE3xFLAG chimeric proteins. For each co-infection combination, we confirmed that nearly $100 \%$ of the inclusions were positive for each construct by immuno-fluorescence (data not shown). The lysates were subjected to immuno-precipitation using antiFLAG antibodies and co-immuno-precipitation of IncD-Myc was assayed by western-blot (Figure 6). The IncD/IncE-3xFLAG chimeric proteins were immuno-precipitated as efficiently as IncD-3xFLAG. As observed before (Figures 3, 4) IncD-Myc coimmuno-precipitated with IncD-3xFLAG. In addition, IncDMyc co-immuno-precipitated with two of the IncD/IncE chimeric constructs, IncDDE and IncEDD, but the IncD/IncDDE and $\mathrm{InCD} / \mathrm{IncEDD}$ interactions were not as robust as the one observed with IncD/IncD.

Altogether these results confirmed that chimeric Inc proteins could be engineered and successfully inserted into C. trachomatis 


IncD IncD IncD IncD IncD IncD IncD IncD
Myc
FLAG
FIGURE 4 I IncD-IncD interaction occurs on C.trachomatis inclusion
membrane. HeLa cells were singly infected in the presence of 2 ng/ml with a
strain of C. trachomatis expressing mCherry constitutively and IncD-3xFLAG
(3F) or IncD-Myc under the control of the aTc inducible promoter or co-infected
with these two strains (IncD-3F+IncDMyc Colnf). Lysates from singly and
co-infected cells were collected and a fourth sample was prepared by mixing
equal part of lysates from the singly infected cells (IncD-3F+IncDMyc Mix). The
lysates were immunoprecipitated with anti-FLAG M2 beads. A portion of the
cell lysate (Left Panel, Lysate) and the immunoprecipitated proteins (Right
Panel, IP) were separated by SDS-PAGE and analyzed by immunoblot with
antibodies against Myc (Top Panels) and FLAG (Bottom Panels).
Inct

inclusion membrane and could be used to dissect the molecular mechanisms driving Inc/Inc protein interaction. Our investigation of $\mathrm{IncD}$ self-interaction revealed that although IncD/IncE chimeric proteins containing the $\mathrm{N}$-terminus and hydrophobic domain (DDE) or the hydrophobic domain and the C-terminus (EDD) of IncD were able to interact with IncD, it appears that the full length IncD protein is required for efficient self-interaction.

\section{DISCUSSION}

Inclusion membrane proteins are specific to Chlamydia and are strategically positioned to mediate inclusion interaction with host factors and organelles. However, little is known about Inc proteins interacting partners and/or function. Inc proteins are difficult to study because of their inherent biochemical properties. Their large hydrophobic domain complicates protein purification in a native and soluble state and limits subsequent in vitro studies to soluble domains (Ronzone and Paumet, 2013; Ronzone et al., 2014). Up until recently, in the absence of genetic tools to manipulate Chlamydia, studies have focused on over-expressing Inc proteins in mammalian cells (Rzomp et al., 2006; Derre et al., 2011; Mital et al., 2013; Mirrashidi et al., 2015) or using two-hybrid system in yeast (Scidmore and Hackstadt, 2001; Rzomp et al., 2006; Lutter et al., 2013; Mital et al., 2015) or bacteria (Gauliard et al., 2015). While these studies have led to the identification of Inc proteins interacting partners and shed light on the putative function of some Inc proteins, the big caveat of these studies is that the Inc proteins were not studied in the natural context of the inclusion membrane, potentially leading to interaction artifacts due to the absence of lipids and/or bacterial and host proteins that would normally constitute the natural environment surrounding the Inc proteins.

With the development of Chlamydia genetic tools (Wang et al., 2011; Sixt and Valdivia, 2016), it is now possible to express Inc proteins of interest from the bacteria and to study their roles in the context of the inclusion membrane (Agaisse and Derre, 2014; Bauler and Hackstadt, 2014; Kokes et al., 2015; Weber et al., $2015,2016)$. We have previously used this method to further investigate IncD/CERT interaction in the context of the infection and to confirm the direct role of IncD in CERT recruitment to the inclusion membrane (Derre et al., 2011; Agaisse and Derre, 2014).

In the current study, we sought to follow up on a bacterial two-hybrid study suggesting that IncD may interact with itself and with a subset of Inc proteins (Gauliard et al., 2015). To probe for Inc/Inc interaction in the context of $C$. trachomatis infection, we took advantage of the fact that nascent inclusions originating from bacteria expressing two different Inc protein variants will eventually undergo fusion resulting in inclusions that display both Inc proteins on the surface of their membranes.

We were able to detect IncD/IncD interaction indicating that IncD may act as a dimer or an oligomer. Our data are also consistent with IncD self-interaction occurring only when the protein is inserted into the inclusion membrane. In the light of our previous data, showing that IncD interacts with the $\mathrm{PH}$ domain of CERT (Derre et al., 2011; Agaisse and Derre, 2014), one could envision that IncD dimerization or oligomerization may enhance the efficacy of CERT recruitment to the inclusion.

In our model system, we did not detect the IncD/CTL0314 and IncD/CTL0475 interactions previously observed in a bacterial two-hybrid system. There are two possible explanations to this discrepancy: (1) we failed to detect these interactions under our experimental set up or (2) the interactions observed using the bacterial two-hybrid system were not physiologically relevant. These results emphasize the need of validating any Inc/Inc interaction by different approaches, preferably in the context of infected cells.

If IncD self-interacts, what are the domains driving this interaction? IncD is a $15 \mathrm{kDa}$ protein that can be separated into three major domains: A N-terminal domain (aa139) that contains the Type III secretion signal, a central hydrophobic domain (aa40-91) responsible for inclusion membrane anchoring and a C-terminal domain (aa92-146). Both the $\mathrm{N}$ - and C-terminal domains are predicted to face the cytosol. To study IncD variants, that are potentially defective for $\mathrm{IncD} / \mathrm{IncD}$ interaction, in the context of the inclusion membrane, these variants should retain the Type III secretion signal and the hydrophobic domain for inclusion membrane localization.

Given that IncD is a fairly small protein, the type of truncated variants that can be generated and studied is therefore limited. We did generate a variant of $\mathrm{IncD}$ that lacked the C-terminal domain, but although the corresponding protein was expressed in C. trachomatis, it failed to display strong inclusion localization (data not shown). 
A
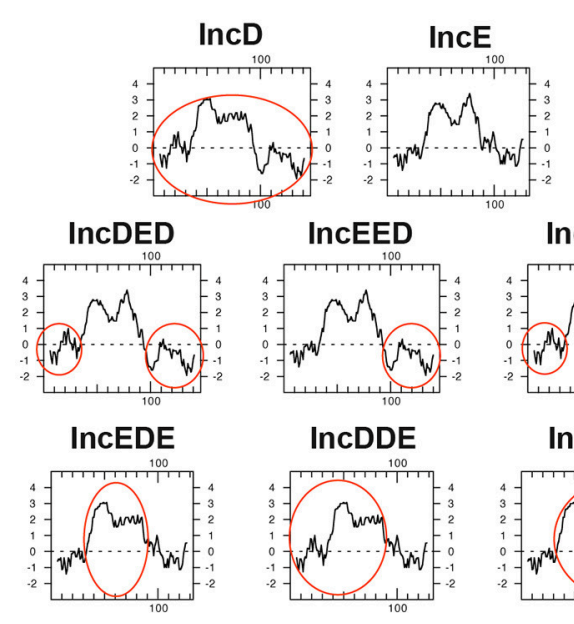

B

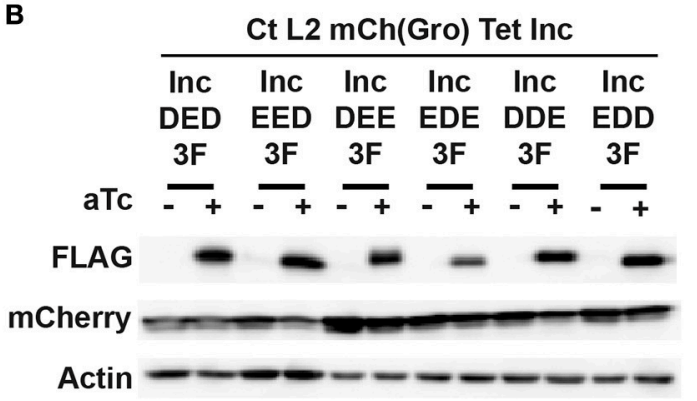

C

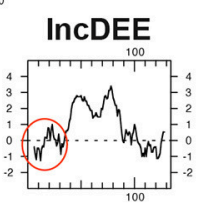
IncDDE
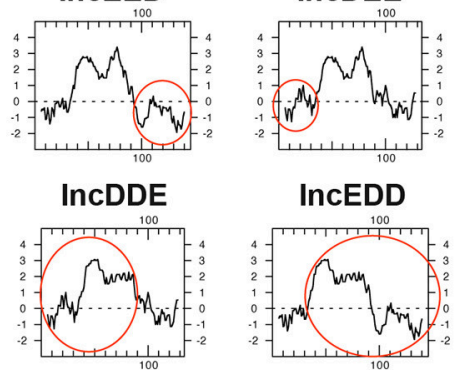

(n)
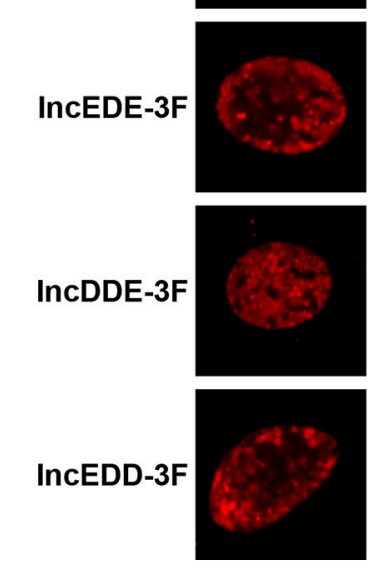
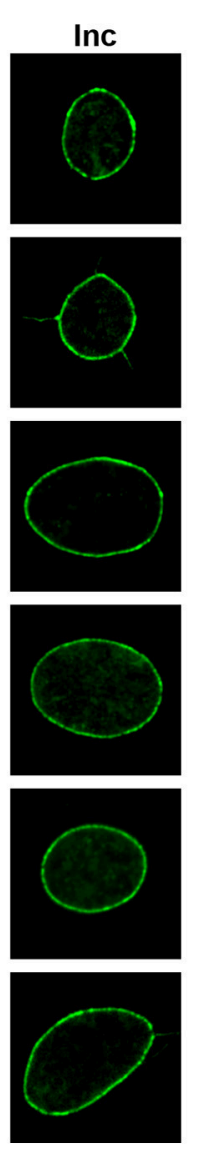
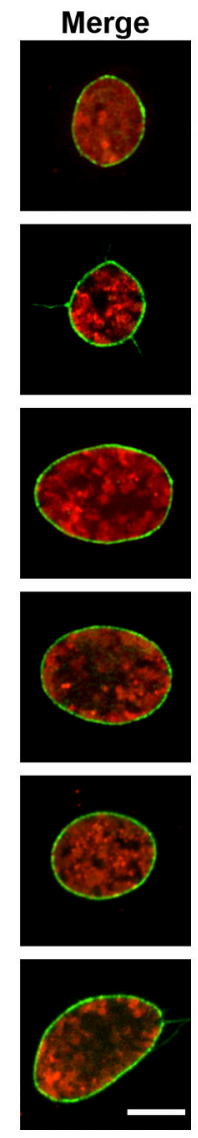

FIGURE 5 | IncD/IncE chimeric proteins localize to C. trachomatis inclusion membrane. (A) Hydropathy plots of IncD, IncE, and the IncD/IncE chimeric proteins used in this study. The red circles indicate domains of IncD. (B) Immuno-blot of cell lysates from HeLa cells infected with strains of $C$. trachomatis expressing mCherry constitutively and the indicated IncD/IncE-3xFLAG (3F) chimeric proteins under the control of the aTc inducible promoter. The cells were infected for $24 \mathrm{~h}$ in the absence (-aTc) or in the presence (+aTc) of $2 \mathrm{ng} / \mathrm{ml}$ aTc and immuno-blot of the corresponding lysates were probed using antibodies against FLAG, mCherry, and Actin. (C) Confocal micrographs of inclusions of the C. trachomatis strains listed in (B). The cells were infected in the presence of $2 \mathrm{ng} / \mathrm{ml}$ aTc, fixed $24 \mathrm{~h}$

post-infection, immunostained with anti-FLAG antibodies and imaged using a confocal microscope. A single plane crossing the middle of the inclusion is shown. The left panels correspond to the bacteria (Chlamydia, red) and the middle panels to the FLAG signal of the IncD/IncE chimera (Inc, green). The merge is shown on the right. Scale bar: $10 \mu \mathrm{m}$.

We therefore turned to chimeric Inc proteins that contained various permutations of the $\mathrm{N}$-, C-terminal, and hydrophobic domains of IncD and IncE, another small Inc protein that did not interact with IncD. Only two chimeras were able to interact with IncD. The combination of the N-terminal and hydrophobic domains of IncD (DDE) led to weak interaction and the hydrophobic domain combined to the C-terminal domain (EDD) led to a slightly more efficient binding. The efficacy of binding of these two chimeric proteins was however weaker than the one observed with the fulllength protein. Our data suggest that the full-length IncD protein is required for dimerization and/or oligomerization. Alternatively, the hydrophobic domain may be sufficient to mediate IncD self-interaction but optimal self-interaction may require additional amino acids that were not included in our constructs, to accommodate optimal self-interaction. Finally, it is possible that, although the hydrophobic domain is sufficient to mediate IncD self-interaction, oligomers formed less efficiently between un-identical IncD units. Testing the self-interaction of the IncDDE and IncEDD chimera could address this question.

Our data suggest that, if not the full-length protein, a large portion of IncD is required for self-interaction and potentially for function. This would be in contrast with IncE, which has been proposed to be a monomer (Gauliard et al., 2015) and for which the C-terminal domain is sufficient for binding the PX domain of SNX5 and SNX6 (Mirrashidi et al., 2015). Altogether, one could envision that some Inc proteins, such as IncE, act as monomers and have distinct domains dedicated to interaction with host factors, while others, like IncD, require oligomerization of the full-length protein to efficiently recruit their host target to the inclusion membrane.

With the recent advances in Chlamydia genetics, it is now possible to investigate the role of $C$. trachomatis inclusion 


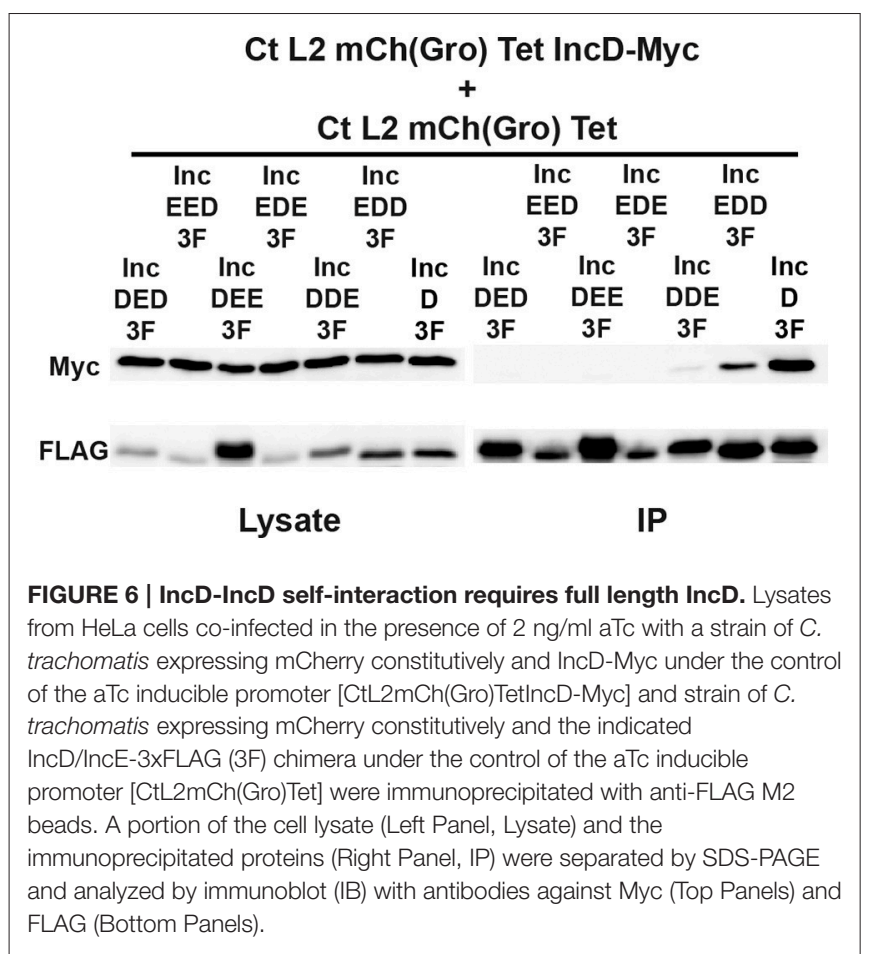

membrane proteins in the context of an infected cell. The co-infection system described here and the use of chimeric Inc proteins, together with the generation of C. trachomatis strains lacking Inc proteins of interest will be powerful tools to assay not only Inc protein functions, but also the molecular mechanisms by which Inc proteins interact with themselves or with host factors, ultimately leading to a better understanding of Chlamydia life cycle and interaction with the mammalian host.

\section{AUTHOR CONTRIBUTIONS}

YH: Performed the experiments, Analyzed the data. ID: Designed the experiments, Performed the experiments, Analyzed the data, Wrote the manuscript.

\section{FUNDING}

NIH NIAID grant R01AI101441 to ID.

\section{ACKNOWLEDGMENTS}

We would like to thank Hervé Agaisse for constructive discussion and critical reading of the manuscript.

\section{SUPPLEMENTARY MATERIAL}

The Supplementary Material for this article can be found online at: http://journal.frontiersin.org/article/10.3389/fcimb. 2017.00079/full\#supplementary-material

Supplementary Figure 1 | Inclusion localization of CTL0314- and CTL0475-3xFLAG. Confocal micrographs of inclusions of $C$. trachomatis strains expressing mCherry constitutively and CTL0314-3xFLAG (Top panels) or CTL0475-3xFLAG (Bottom panels) under the control of the aTc inducible promoter. The cells were infected in the presence of $2 \mathrm{ng} / \mathrm{ml}$ aTc, fixed $24 \mathrm{~h}$ post-infection, immunostained with anti-FLAG (Yellow), and anti-IncA (Blue) antibodies and imaged using a confocal microscope. A single plane crossing the middle of the inclusion is shown. The left panels correspond to the bacteria (Chlamydia, red). The merge is shown on the right. Scale bar: $10 \mu \mathrm{m}$.

Supplementary Table 1 | Primers used in this study.

\section{REFERENCES}

Aeberhard, L., Banhart, S., Fischer, M., Jehmlich, N., Rose, L., Koch, S., et al. (2015). The proteome of the isolated Chlamydia trachomatis containing vacuole reveals a complex trafficking platform enriched for retromer components. PLoS Pathog. 11:e1004883. doi: 10.1371/journal.ppat.1004883

Agaisse, H., and Derre, I. (2013). A C. trachomatis cloning vector and the generation of $C$. trachomatis strains expressing fluorescent proteins under the control of a C. trachomatis promoter. PLoS ONE 8:e57090. doi: 10.1371/journal.pone.0057090

Agaisse, H., and Derre, I. (2014). Expression of the effector protein IncD in Chlamydia trachomatis mediates recruitment of the lipid transfer protein CERT and the endoplasmic reticulum-resident protein VAPB to the inclusion membrane. Infect. Immun. 82, 2037-2047.doi: 10.1128/IAI.01530-14

Bannantine, J. P., Griffiths, R. S., Viratyosin, W., Brown, W. J., and Rockey, D. D. (2000). A secondary structure motif predictive of protein localization to the chlamydial inclusion membrane. Cell. Microbiol. 2, 35-47. doi: 10.1046/j.1462-5822.2000.00029.x

Bauler, L. D., and Hackstadt, T. (2014). Expression and targeting of secreted proteins from Chlamydia trachomatis. J. Bacteriol. 196, 1325-1334. doi: 10.1128/JB.01290-13

Dehoux, P., Flores, R., Dauga, C., Zhong, G., and Subtil, A. (2011). Multi-genome identification and characterization of chlamydiae-specific type III secretion substrates: the Inc proteins. BMC Genomics 12:109. doi: $10.1186 / 1471-2164-12-109$

Derre, I., Pypaert, M., Dautry-Varsat, A., and Agaisse, H. (2007). RNAi screen in Drosophila cells reveals the involvement of the Tom complex in Chlamydia infection. PLoS Pathog. 3:e030155. doi: 10.1371/journal.ppat.0030155

Derre, I., Swiss, R., and Agaisse, H. (2011). The lipid transfer protein CERT interacts with the Chlamydia inclusion protein IncD and participates to ER-Chlamydia inclusion membrane contact sites. PLoS Pathog. 7:e1002092. doi: 10.1371/journal.ppat.1002092

Elwell, C., Mirrashidi, K., and Engel, J. (2016). Chlamydia cell biology and pathogenesis. Nat. Rev. Microbiol. 14, 385-400. doi: 10.1038/nrmicro.2016.30

Gauliard, E., Ouellette, S. P., Rueden, K. J., and Ladant, D. (2015). Characterization of interactions between inclusion membrane proteins from Chlamydia trachomatis. Front. Cell. Infect. Microbiol. 5:13. doi: 10.3389/fcimb.2015.00013

Hackstadt, T., Scidmore-Carlson, M. A., Shaw, E. I., and Fischer, E. R. (1999). The Chlamydia trachomatis IncA protein is required for homotypic vesicle fusion. Cell. Microbiol. 1, 119-130. doi: 10.1046/j.1462-5822.1999.00012.x

Kokes, M., Dunn, J. D., Granek, J. A., Nguyen, B. D., Barker, J. R., Valdivia, R. H., et al. (2015). Integrating chemical mutagenesis and whole-genome sequencing as a platform for forward and reverse genetic analysis of Chlamydia. Cell Host Microbe 17, 716-725. doi: 10.1016/j.chom.2015.03.014

Li, Z., Chen, C., Chen, D., Wu, Y., Zhong, Y., and Zhong, G. (2008). Characterization of fifty putative inclusion membrane proteins encoded in the Chlamydia trachomatis genome. Infect. Immun. 76, 2746-2757. doi: 10.1128/IAI.00010-08

Lutter, E. I., Barger, A. C., Nair, V., and Hackstadt, T. (2013). Chlamydia trachomatis inclusion membrane protein CT228 recruits elements of the 
myosin phosphatase pathway to regulate release mechanisms. Cell Rep. 3, 1921-1931. doi: 10.1016/j.celrep.2013.04.027

Lutter, E. I., Martens, C., and Hackstadt, T. (2012). Evolution and conservation of predicted inclusion membrane proteins in chlamydiae. Comp. Funct. Genomics 2012:362104. doi: 10.1155/2012/362104

Mirrashidi, K. M., Elwell, C. A., Verschueren, E., Johnson, J. R., Frando, A., Von Dollen, J., et al. (2015). Global mapping of the inc-human interactome reveals that retromer restricts Chlamydia infection. Cell Host Microbe 18, 109-121. doi: 10.1016/j.chom.2015.06.004

Mital, J., Lutter, E. I., Barger, A. C., Dooley, C. A., and Hackstadt, T. (2015). Chlamydia trachomatis inclusion membrane protein CT850 interacts with the dynein light chain DYNLT1 (Tctex1). Biochem. Biophys. Res. Commun. 462, 165-170. doi: 10.1016/j.bbrc.2015.04.116

Mital, J., Miller, N. J., Dorward, D. W., Dooley, C. A., and Hackstadt, T. (2013). Role for chlamydial inclusion membrane proteins in inclusion membrane structure and biogenesis. PLoS ONE 8:e63426. doi: 10.1371 /journal.pone.0063426

Mital, J., Miller, N. J., Fischer, E. R., and Hackstadt, T. (2010). Specific chlamydial inclusion membrane proteins associate with active Src family kinases in microdomains that interact with the host microtubule network. Cell. Microbiol. 12, 1235-1249. doi: 10.1111/j.1462-5822.2010.01465.x

Moore, E. R., and Ouellette, S. P. (2014). Reconceptualizing the chlamydial inclusion as a pathogen-specified parasitic organelle: an expanded role for Inc proteins. Front. Cell. Infect. Microbiol. 4:157. doi: 10.3389/fcimb.2014.00157

Moulder, J. W. (1991). Interaction of chlamydiae and host cells in vitro. Microbiol. Rev. 55, 143-190.

Mueller, K. E., Plano, G. V., and Fields, K. A. (2014). New frontiers in type III secretion biology: the Chlamydia perspective. Infect. Immun. 82, 2-9. doi: 10.1128/IAI.00917-13

Ridderhof, J. C., and Barnes, R. C. (1989). Fusion of inclusions following superinfection of HeLa cells by two serovars of Chlamydia trachomatis. Infect. Immun. 57, 3189-3193.

Ronzone, E., and Paumet, F. (2013). Two coiled-coil domains of Chlamydia trachomatis IncA affect membrane fusion events during infection. PLoS ONE 8:e69769. doi: 10.1371/journal.pone.0069769

Ronzone, E., Wesolowski, J., Bauler, L. D., Bhardwaj, A., Hackstadt, T., and Paumet, F. (2014). An alpha-helical core encodes the dual functions of the chlamydial protein IncA. J. Biol. Chem. 289, 33469-33480. doi: $10.1074 /$ jbc.M114.592063
Rzomp, K. A., Moorhead, A. R., and Scidmore, M. A. (2006). The GTPase Rab4 interacts with Chlamydia trachomatis inclusion membrane protein CT229. Infect. Immun. 74, 5362-5373. doi: 10.1128/IAI.00539-06

Schachter, J. (1999). "Infection and disease epidemiology," in Chlamydia: Intracellular Biology, Pathogenesis, and Immunity, ed R. S. Stephens (Wahsington, DC: American Society for Microbiology), 139-170.

Scidmore, M. A., and Hackstadt, T. (2001). Mammalian 14-3-3beta associates with the Chlamydia trachomatis inclusion membrane via its interaction with IncG. Mol. Microbiol. 39, 1638-1650. doi: 10.1046/j.1365-2958.2001. 02355.x

Sixt, B. S., and Valdivia, R. H. (2016). Molecular genetic analysis of Chlamydia species. Annu. Rev. Microbiol. 70, 179-198. doi: 10.1146/annurev-micro-102215-095539

Stephens, R. S., Kalman, S., Lammel, C., Fan, J., Marathe, R., Aravind, L., et al. (1998). Genome sequence of an obligate intracellular pathogen of humans: Chlamydia trachomatis. Science 282, 754-759. doi: $10.1126 /$ science.282.5389.754

Wang, Y., Kahane, S., Cutcliffe, L. T., Skilton, R. J., Lambden, P. R., and Clarke, I. N. (2011). Development of a transformation system for Chlamydia trachomatis: restoration of glycogen biosynthesis by acquisition of a plasmid shuttle vector. PLoS Pathog. 7:e1002258. doi: 10.1371/journal.ppat.1002258

Weber, M. M., Bauler, L. D., Lam, J., and Hackstadt, T. (2015). Expression and localization of predicted inclusion membrane proteins in Chlamydia trachomatis. Infect. Immun. 83, 4710-4718. doi: 10.1128/IAI.01075-15

Weber, M. M., Noriea, N. F., Bauler, L. D., Lam, J. L., Sager, J., Wesolowski, J., et al. (2016). A functional core of IncA is required for Chlamydia trachomatis inclusion fusion. J. Bacteriol. 198, 1347-1355. doi: 10.1128/JB.00 933-15

Conflict of Interest Statement: The authors declare that the research was conducted in the absence of any commercial or financial relationships that could be construed as a potential conflict of interest.

Copyright $\odot 2017$ Han and Derré. This is an open-access article distributed under the terms of the Creative Commons Attribution License (CC BY). The use, distribution or reproduction in other forums is permitted, provided the original author(s) or licensor are credited and that the original publication in this journal is cited, in accordance with accepted academic practice. No use, distribution or reproduction is permitted which does not comply with these terms. 